\title{
Mapping Anisotropic Landscape for Understanding Underlying Spatial Structure of Air Pollution
}

\author{
Chen-Hsuan $\mathrm{Tu}^{\mathrm{a}}{ }^{*}$ and Tzai-Hung Wen ${ }^{\mathrm{a}}$ \\ a National Taiwan University,r07228005@ntu.edu.tw,wenthung@ntu.edu.tw \\ * Corresponding author
}

Keywords: Anisotropic, Spatial Interpolation, Air Quality

\begin{abstract}
:
Urban air pollution problem has become a huge threat to human health in the most developing and developed countries. Therefore, monitoring air quality with high spatial and temporal resolutions is an important issue. There are two different approaches to mapping street-level distributions of air quality in time and space. One is mathematical approach, which uses numerical methods to calculate the concentration of air pollutants in each space-time grid through considering chemical transport, wind field, terrain morphology and other parameters which affect the direction and intensity of dispersion. This approach is limited by intensively computational process, so most of studies used either rough spatial grid resolution for representing large-scale regions or detailed grid resolution for small-scale areas. Numerical models with rough grid resolution could not capture detailed physical interactions in the micro-environment. The other approach is statistical approach, which used spatial interpolation techniques, such as inverse distance weighting (IDW) and Kriging methods, or established regression models, such as land-use regression (LUR), for deriving concentrations of air pollution from remote sensing or ground-level station sensor data. This approach is assumed linear associations with environmental factors and isotropic distance-decayed phenomena, which also ignores complex physical interactions.
\end{abstract}

Spatial distribution of air pollution could be affected by directional background factors, such as wind fields, surface relief and so on. The spatial effects of these physical factors are not isotropic. However, recent studies used statistical modelling approaches are based on isotropic assumptions and did not consider directional variations of these factors on air quality. The purpose of the study is to develop an innovative statistical approach to measure directional effects on air quality with spatial heterogeneity. We produces anisotropic landscapes of directional fields for identifying major directions for each space-time grid through EPA's monitoring station data to visualize space-time trend of air quality changing with directions. This study provides significant insight for understanding spatial structures behind air pollution distributions influenced by directional physical factors. 